\title{
A Survey on Privacy Preserving Techniques in WSN
}

\author{
Snehalata K.Funde \\ Department of Computer Engineering \\ Faculty of Engineering \\ University of Pune, Pune
}

\author{
A.D.Gujar \\ Department of Computer Engineering \\ Faculty of Engineering \\ University of Pune, Pune
}

\begin{abstract}
A great part of the existing tackle Wireless Sensor Networks (WSN) has concentrated on tending to the force and computational asset demands of WSN by the configuration of particular steering, MAC, and cross-layer conventions. As of late, there have been increased privacy concerns over the data gathered by and transmitted through WSN. The remote transmission needed by a WSN, and the self-composing nature of its structural engineering, makes privacy assurance for WSN a particularly testing issue. This paper gives a stateof-art review of privacy-preserving strategies for WSN. Specifically, we audit two fundamental classes of privacypreserving strategies for ensuring two sorts of private data, data-turned and connection situated privacy, separately. We likewise talk about a number of essential open challenges for future research. Our trust is that this paper sheds some light on a productive course of prospective research for privacy conservation in WSN.
\end{abstract}

\section{General Terms \\ Privacy, WSN}

Keywords

WSN, MAC, Data privacy

\section{INTRODUCTION}

As of late, wireless sensor systems (WSNs) have drawn significant consideration from the exploration neighborhood on issues going from hypothetical research to viable requisitions. Uncommon qualities of WSNs, for example asset requirements on vigor and computational force, have been generally characterized and broadly mulled over. What has accepted less consideration, be that as it may, is the basic security concern on data being gathered, transmitted, and broke down in a WSN. Such private data of concern may incorporate payload information gathered by sensors and transmitted through the system to an unified information handling server. Case in point, a patient's pulse, sugar level also other basic signs are generally of discriminating protection concern the point when screened by a restorative WSN which transmits the information to a remote healing center or specialist's office. Protection concerns might additionally go out past information content and might center on connection data, for example the area of a sensor launching information correspondence. Note that an alarm correspondence beginning from a patient's heart screen in the restorative WSN is sufficient for an enemy to deduce that the persistent experiences heart issue. Adequate countermeasure against the revelation of both information and setting arranged private data is a vital essential for the expansive requisition of WSNs to certifiable provisions [1]. Security assurance has been broadly examined in different fields identified with WSN, for example wired and remote systems administration, databases and information mining. In any case, the emulating inalienable characteristics of WSNs present interesting challenges for security conservation in WSNs, and anticipate the existing systems from being straightforwardly transplanted:

- Nature's turf: Sensors might need to be conveyed to an environment wild by the safeguard, for example a front line, empowering an enemy to start physical strike to catch sensor hubs or send fake ones. Subsequently, a foe might recover private keys utilized for secure correspondence and unscramble any correspondence spied by the foe.

- Sensor-hub asset demands: An electric cell fueled sensor hub for the most part has intense requirements on its capability to store, process, and transmit the sensed information. Accordingly, the computational multifaceted nature and asset utilization of open key figures is normally recognized inadmissible for WSNs. This presents extra challenges for protection conservation.

- Topological demands: The constrained correspondence go of sensor hubs in a WSN requires different bounces to transmit information from the source to the base station. Such a multi-bounce plan requests diverse hubs to take various movement stacks. Specifically, a hub closer to the base station (i.e., information gathering and handling server) needs to hand-off information from hubs further far from base station notwithstanding transmitting its own produced information, prompting higher transmission rate. Such an uneven system movement design carries noteworthy tests to the security of setting arranged protection data. Especially, if an enemy holds the capability of worldwide activity investigation, watching the movement designs of distinctive hubs over the entire system, it can effortlessly distinguish the sink and trade off setting security, on the other hand even control the sink hub to block the correct working of the WSN.

The special tests for security safeguarding in WSNs call for the advancement of successful protection protecting strategies. In this paper, we furnish a state-of-the-craft study of existing protection protecting methods in WSNs. We survey two fundamental classes of security protecting methods for securing two sorts of private data, information arranged and setting turned security, separately. In the class of information security, we predominantly examine how to empower the total of sensed information without maltreating the protection of the information being gathered and insurance the advancement of the field of Security of information inquiry launched by clients of the system. For setting based protection, we examine the security of area security and fleeting private data. [2]. Last yet not the slightest, we examine some intriguing and testing open issues on this point, which are relied upon to shed light on a productive course of prospective research on security protection in WSNs. 


\section{RELATED WORK}

Scrutinize on issues identified with WSNs requires multidisciplinary studies crossing systems administration, databases, circulated figuring, and so forth. To legitimately comprehend the tests of protection conservation in WSNs and the systems important to address such provokes, it is essential to first analyze the protection issues and security saving procedures in such identified fields as databases, information mining furthermore remote systems, which we quickly survey as takes after. In the field of database and information mining, security concerns may emerge from three sorts of frameworks The first is a data imparting framework which includes two or all the more commonly untrusted gatherings. The target is to certification that no private data past the base vital is revealed throughout data offering. Cryptographic secure multi-party reckoning strategies are generally utilized for this sort of frameworks. The second is an information accumulation framework where one brought together information collector/analyzer gathers and mines information from different dispersed information suppliers. Arbitrary annoyance strategies are generally connected to securing protection in these frameworks. The third sort is an information distributed framework, the target of which is to distribute information to back information systematic requisition without trading off the namelessness of singular information managers. K-secrecy and diversity based calculations are proposed for security assurance in these frameworks [3]. Security issues have additionally been broadly contemplated in the realm of non specific systems administration. Area protection is of specific concern with the pervasive improvement of progressed remote mechanism, such as PDA, and with the coming of area based administration (LBS). In a LBS framework, a client holding a remote apparatus inquiries about the LBS server to get to the closest restaurant or healing facility of the client regardless, the client might not energetically reveal his/her genuine area. To address such area security concerns, Anonymizer, a believed unbiased gathering based skeleton, was proposed. With this system, a client first sends his/her area to the incorporated anonymizer which then inquiries the LBS server with not the client's true area yet a shrouding locale which blankets the client as well as a number of different clients. This procedure anticipates the LBS server from recognizing one client from numerous others. Notwithstanding, it is unrealistic to be handy for two explanations: First, it is definitely not sensible to expect the presence of a trustable third party. Second, regardless of the fact that such a believed unbiased gathering exists, it makes a solitary purpose ofdisappointment for the framework on the grounds that if the unbiased gathering is bargained, the security protection over the entire framework will totally crumple. To evacuate the prerequisite of a believed unbiased gathering, a private-data recovery based procedure was proposed. In any case, this system likewise experiences huge calculation and correspondence overhead. Plus the administer revelation of client's area from question payload; movement stream data might likewise rupture area security. Specifically, the server might pinpoint the area of a client in view of the client's IP address. Keeping in mind the end goal to furnish movement stream classifiedness, To is composed, as the second era onion steering, and turns into a prevalent unknown correspondence system which comprises of many Tor switches to transfer client movement to or from LBS s.[3]

\subsection{Data Oriented Privacy Protection.}

Information situated protection assurance keeps tabs on securing the security of information substance. Here "information" alludes to not just sensed information gathered inside a WSN additionally inquiries postured to a WSN by clients. In the aforementioned therapeutic WSN sample, private data might incorporate temperature furthermore pulse gathered from the WSN, or questions on these imperative signs postured to the WSN. There are two sorts of foes which might bargain information turned security. One is an outside foe which listens in the information correspondence between sensor hubs in a WSN [1], [2], [3]. This kind of enemies could be successfully safeguarded against utilizing the conventional systems of cryptographic encryption and confirmation. The second sort is an inward enemy which is likewise a partaking hub of the WSN, yet has been caught and controlled by vindicating the elements to bargain private data. Since a partaking hub is permitted to unscramble information lawfully, the universal encryption and confirmation systems might never again be successful. Consequently, the fundamental test for securing information situated protection is to forestall an interior foe from trading off the private data, while supporting the typical operation of the WSN.

\subsection{Context Oriented Privacy Protection.}

Connection arranged protection insurance concentrates on ensuring logical data, for example the area and timing data of activity transmitted in a WSN. Area protection concerns may emerge for such exceptional sensor hubs as the information source and the base station. As we said in Section 1, an enemy with information of the area of the information source or base station area may have the ability to construe the substance of the information being transmitted on the other hand crush the sensor system. Timing protection, then again, concerns the time the point when touchy information is made at information source, gathered by a sensor hub and transmitted to the base station. This sort of protection is additionally of essential essentialness, particularly in the portable target following requisition of WSNs, on the grounds that an enemy with information of such timing data might have the ability to pinpoint the nature and area of the followed focus without studying the information being transmitted in the WSN. Moreover, the enemy may have the ability to foresee the moving way of the portable focus sometime to come, abusing the protection of the target. Like information arranged protection, setting situated security might additionally be undermined by both outside and inside enemies [4],[5]. Regardless, existing research has for the most part centered on shielding against outside foes, in light of the fact that such foes may have the ability to bargain setting protection effectively by screening remote correspondence. Inside the classification of outside foes, one can further characterize enemies into two classifications, neighborhood assaulters and worldwide aggressors, taking into account the quality of assaults a foe is equipped for starting. Neighborhood assailants can just screen a neighborhood the scope region of a WSN, and thusly need to break down movement jump bybounce to bargain movement connection data. Then again, a worldwide aggressor has the capacity (e.g., a high-pick up receiving wire) of observing the worldwide activity in a WSN. One can see that a worldwide assaulter is much stronger than a neighborhood one. We might want to comment that the grouping of connection situated protection into the two classifications of area furthermore timing protection just reflects the present state-of-the art, furthermore ought not be treated as an extensive grouping. Setting arranged security likewise enlarges to different concerns, for example the stowing away of time recurrence of correspondence between sensor hubs in a WSN, in light of the fact that such recurrence might uncover data about the movement stream in a WSN [6], [7]. In the aforementioned restorative WSN, realizing that a 
patient's circulatory strain sensor is having visit correspondence with its neighboring hubs is sufficient for a foe to gather that the patient may be enduring from high or low blood vessel pressure. Which Mutation Testing has gave an approach to evaluate the nature of test suites; however there has been relatively finish up enhancing the test suites, based on the co- partnered mutation investigation. We want that, in future, there will be significantly more work that looks to utilize high caliber mutants as a foundation for creating high caliber test information. Nonetheless, at present, commonsense programming test information era for mutation test sufficiency remains an uncertain problem.[8][9]

\section{HURDLES TO OVERCOME}

While some work has been proposed in protection security in WSNs, there are still numerous open scrutinize issues in need of prospective exploration. Here we record some critical ones.

- In the situation of Panda Hunting, the existing work just addresses frameworks with a solitary portable target (panda). An intriguing open issue might be to handle the cases with numerous portable targets.

- A noteworthy test is to viably ensure the area of a portable base station. In actuality, by instinct, the portability of base station should furnish some assurance to its area security against outside assault, be that as it may, it needs to upgrade its area to all or part of hubs in the system so they could send information towards it, which certainly makes more opportunity to stick focus it through inside strike. Appropriately, how to secure the area security of a versatile base station as well as ensuring the framework practicality is test of essential criticalness. [10]

- In, re-encryption strategy is proposed to change the presence of information with a specific end goal to kill the likelihood of deciphering base station by following one information bundle in a thick system. At the same time in an inadequate WSN, it is still profoundly likely for foe to deduce the moving of one information bundle without the irritating of thick information activity, notwithstanding disguising the information bundle by encrypting it. Accordingly, how to make re-encryption strategy work viably in an inadequate system is still testing.

- Considering the guaranteeing provisions of WSNs to our genuine physical planet, new sorts of relevant security data might turn out, which are conceivably joined with such potential research fields as informal organization. We are anticipating the happening to them also their contraventions which steadily improve the protection conservation in WSNs.

\section{CONCLUSION}

In this paper, we have introduced a state-of-the-symbolization overview on security safeguarding strategies in WSNs. We talked about the existing security safeguarding strategies in two classes, which address information situated and setting arranged security concerns. The information turned strategies address the assurance of private information transmitted in the WSN and that of delicate inquiries executed. Setting situated methods, then again, ensure the area of the information source and the base station too as the timing of the era and transmission of delicate information. Likewise, we endeavored to analyze the existing systems regarding such measurements as security, exactness, delay furthermore force utilization. Through extensive investigation of security issues in WSNs, going from issue definitions to the existing systems, we portrayed a complete picture of the state-of-thesymbolization on protection conservation in WSNs. Besides, in light of the existing work, we recorded various open issues which might interest the investment of specialists for future work. It is our trust that this paper sheds lights on a productive heading of prospective research on security conservation for WSNs.

\section{ACKNOWLEDGEMENT}

I take this opportunity to express my deepest thanks and gratitude to head of the department Prof.S.M.Chaware for his constant support encouragement and suggestions to shape this survey in a systematic way. I am also greatly indebted to Prof.A.D.Gujar for his inspiring guidance, encouragement and constructive criticism at every stage which helped in the preparation of the survey. Finally I would like to thank all my staff members for they were constantly available and helpful to me in all the needs to prepare this survey.

\section{REFERENCES}

[1] R.Agrawal ,R.Srikan ,Prvacy preserving Data mining in proceedings of the 2008 ACM SIGMOD on mamnagement of data, Dallas TX USA MAY 15-18 2000, pp 439-450.

[2] G. Ghinita, P. Kalnis, A. Khoshgozaran, C. Shahabi, Tan, Kian-Lee, Private queries in location based services: anonymizers are not necessary, in: Proceedings of the 2008 ACM SIGMOD international conference on Management of data, Vancouver, Canada, 2008, pp. 121-132.

[3] LF Akyilidiz, W.Su, Y Shankarsubramaniam, E.Cayirci, Wireless sensor networks: a survey, computer networks 38(4) 2002 (393-422).

[4] R. Dingledine, N. Mathewson, P. Syverson, Tor: the secondgeneration Onion router, in: Proceedings of the 13th USENIX Security Symposium, 2004

[5] J. Deng, R. Han, S. Mishra, Intrusion tolerance and antitraffic analysis strategies for wireless sensor networks, in: IEEE International Conference on Dependable Systems and Networks (DSN 2004), Florence, Italy, 28 June-1 July 2004, pp. 637-646.

[6] J. Deng, R. Han, S. Mishra, Countermeasures against traffic analysis attacks in wireless sensor networks, in: First International Conference on Security and Privacy for Emerging Areas in Communications Networks (SecureComm) 2005, September 2005, pp. 113-126.

[7] J. Deng, R. Han, S. Mishra, Decorrelating wireless sensor network traffic to inhibit traffic analysis attacks, Pervasive and Mobile Computing Elsevier 2 (2) (2006) 159-186.

[8] E.D. Cristofaro, J. Bohli, D.Westho, FAIR: fuzzy-based aggregation providing in-network Resilience for realtime wireless sensor networks, to appear, in: Proceedings of the Second ACM Conference Wireless Network Security (Wisec), 2009.

[9] M.J. Freedman, K. Nissim, B. Pinkas, Efficient private matching and set intersection, in: Advances in Cryptography, Proceedings of Eurocrypt 2004, 2004, pp. $1-19$.

[10] R.Agrawal, A.Evfimievski, R.Srikant, information sharing across private databases: proceedings of the 2003ACM SIGMOD International conference on management of data, 2003 , pp.86- 97 